\title{
Un aspect de l'anticomanie dans les pays de l'Ouest : histoire des collections grecques, étrusques et phéniciennes en Bretagne et dans les Pays de la Loire
}

\section{Dominique Frere et Marie-Hélène Santrot}

\section{(2) OpenEdition Journals}

Édition électronique

URL : http://journals.openedition.org/abpo/1134

DOI : 10.4000/abpo.1134

ISBN : 978-2-7535-1497-3

ISSN : 2108-6443

\section{Éditeur}

Presses universitaires de Rennes

\section{Édition imprimée}

Date de publication : 20 mars 2005

Pagination : 43-60

ISBN : 978-2-7535-0125-6

ISSN : $0399-0826$

\section{Référence électronique}

Dominique Frere et Marie-Hélène Santrot, « Un aspect de l'anticomanie dans les pays de l'Ouest : histoire des collections grecques, étrusques et phéniciennes en Bretagne et dans les Pays de la Loire ", Annales de Bretagne et des Pays de l'Ouest [En ligne], 112-1 | 2005, mis en ligne le 20 mars 2007, consulté le 19 avril 2019. URL : http://journals.openedition.org/abpo/1134 ; DOI : 10.4000/abpo.1134 


\title{
Un aspect de l'anticomanie dans les pays de l'Ouest : histoire des collections grecques, étrusques et phéniciennes en Bretagne et dans les Pays de la Loire
}

\author{
Dominique FRERE \\ Maître de conférences, Université de Bretagne Sud (Lorient) \\ UMR 8546 - CNRS \\ Marie-Hélène SANTROT \\ Conservateur chargée des collections archéologiques du musée Dobrée (Nantes)
}

Depuis déjà une quinzaine d'années, une collaboration entre les universités et les musées de l'Ouest a permis de faire l'étude scientifique d'un patrimoine important mais souvent méconnu : les bronzes, monnaies, urnes, terres cuites, statues en pierre et vases en céramique et en métal d'origine méditerranéenne. Ces figures de l'Antiquité hantent les réserves de nos musées, témoins modestes ou prestigieux de l'histoire des riches civilisations égyptienne, phénicienne, grecque et étrusque mais témoins aussi d'une grande aventure : celle de l'anticomanie qui marqua une grande partie de l'Europe suite à la découverte de ces civilisations. Cette mode de l'anticomanie est à l'origine de la constitution de nombreuses collections privées qui, par dons et legs, ont intégré les musées souvent à partir de la seconde moitié du XIX ${ }^{\mathrm{e}}$ siècle : un visiteur non prévenu ne voit dans les réserves de ces musées (ou dans les greniers des mairies ${ }^{1}$ !) qu'un bric-àbrac d'objets hétérogènes sans histoire et sans âme. C'est le rôle des conservateurs et des universitaires que de faire revivre ces œuvres dans leur double contexte historique, celui de l'antiquité bien sûr mais aussi celui de l'époque contemporaine. Un programme de recherche a ainsi été élaboré à l'université de Bretagne Sud visant à l'étude scientifique des œuvres antiques considérées d'une part comme des objets archéologiques

1. Voir par exemple la momie égyptienne qui était conservée dans le grenier de la mairie de Vitré : Marie-Christine Budichovsky, "Le cercueil égyptien de Vitré ", Bulletin de la Société Nationale des Antiquaires de France, 1997, p. 40-50. 
apportant des informations historiques sur les techniques et les échanges dans l'Antiquité ${ }^{2}$ et d'autre part comme des éléments de notre patrimoine régional témoins de l'évolution de l'archéologie et de l'histoire des mentalités aux XVIII ${ }^{\mathrm{e}}$ et XIX ${ }^{\mathrm{e}}$ siècles.

C'est toujours le rôle des mêmes scientifiques que de donner sens à ces antiquités expatriées en montrant cette âme qui les anime encore, âme qui avait attiré l'attention des archéologues et collectionneurs des XVIII et $\mathrm{XIX}^{\mathrm{e}}$ siècles et qui passionne actuellement un public nombreux et très diversifié. Le travail de récolement, d'étude scientifique et de publication des collections grecques et étrusques de nombre de musées de l'Ouest a en effet permis l'élaboration d'une importante exposition qui, présentée au musée Dobrée de Nantes, se déplacera jusqu'en 2007 dans neuf sites culturels du Grand Ouest (couvent des Ursulines de Château-Gontier, musée Vendéen de Fontenay-le-Comte, musée de Tessé du Mans, musée des BeauxArts de Vannes, musée Pincé d'Angers, Chapelle Saint-Julien de Laval, musée d'Art et d'Histoire de Cholet et château de Châteaubriant). Le succès public de cette exposition et son retentissement au niveau national ${ }^{3}$ montrent que l'Antiquité, par l'intermédiaire des œuvres d'art et des objets de la vie quotidienne qu'elle a produits, exerce le même pouvoir de fascination qu'au XVIII ${ }^{\mathrm{e}}$ et $\mathrm{XIX}^{\mathrm{e}}$ siècle. Toutefois ce n'est plus, comme à ces époques, l'esprit de curiosité qui est à l'origine de l'étude de ces œuvres et de leur diffusion auprès du grand public, mais la volonté de leur restituer leur identité archéologique et ethnologique, d'en faire des objets scientifiques, des témoins non seulement de l'histoire antique mais aussi de notre histoire récente à l'échelle régionale et à l'échelle européenne.

\section{Les prolégomènes}

Cette histoire commence dans la première moitié du XVIII ${ }^{\mathrm{e}}$ siècle avec comme toile de fond, les publications importantes de Bernard de Montfaucon et, quelques décennies plus tard, de celles du comte de Caylus ${ }^{4}$.

La mode de l'anticomanie qui s'est emparée d'une partie de l'Europe et qui ne fera que se renforcer après les découvertes d'Herculanum et de Pompéi, a bien évidemment des répercussions dans l'Ouest de la France ${ }^{5}$,

2. Ces œuvres donnent lieu à des études d'attribution stylistique et de typologie morphologique qui permettent leur datation et leur attribution géographique. Considérées comme des objets scientifiques, elles ont été intégrées dans un programme de recherches pluridisciplinaires regroupant l'Université de Bretagne-Sud, l'Université d'Angers et celle de Nantes. Des analyses pétrographiques de l'argile et des analyses chromatographiques des contenus (huiles, huiles parfumées et parfums) permettent d'apporter des informations précieuses sur les techniques de fabrication, sur les structures des ateliers, et sur les produits contenus qui représentaient une part importante des échanges dans l'antiquité.

3. Archeologia, n $^{\circ} 411$, mai 2004, p. 36-41.

4. Bernard de Montfaucon, L'Antiquité expliquée et représentée en figures, Paris, 17191724 et Anne Claude Philippe de Tubières, comte de Caylus, Recueil d'antiquités égyptiennes, étrusques, grecques et romaines, Paris, 1752-1764.

5. Roche, Daniel, Le Siècle des Lumières en province. Académies et académiciens 
mais ces échos de l'archéologie méditerranéenne ne vont pas jusqu'à la constitution de grandes collections classiques. Les monuments antiques étaient rares mais pas absents : les sites de Corseul et de Jublains ${ }^{6}$ en particulier, alimentaient, au hasard des "fouilles " et des découvertes fortuites, des petites collections locales ${ }^{7}$ constituées d'objets que l'on considérait tantôt comme gaulois tantôt comme romains. En ce siècle des Lumières, l'intérêt des antiquaires bretons se concentre sur les " souvenirs de la romanité et du passage de César à l'occasion de sa lutte contre les Vénètes ${ }^{8}$ ", et à la différence de ce qui se passe alors dans d'autres régions de France, les élites de l'Ouest n'éprouvent aucun intérêt pour les antiquités de type grec ou étrusque absentes de leur sol. Il faut dire que l'esprit de collectionneur n'est pas une grande tradition dans l'Ouest ${ }^{9}$ qui ne connaît qu'un véritable cabinet de curiosités important, celui du Président ChristophePaul de Robien (1698-1756) ${ }^{10}$. Ainsi, en-dehors de petits cabinets d'histoire naturelle, la Bretagne ne présente guère au XvIII ${ }^{\mathrm{e}}$ siècle de collections d'antiques qui sont rares par exemple à l'Académie royale de Marine de Brest. Cette rareté n'est pas imputable, bien au contraire, à un désintérêt de l'officier de marine pour l'antiquité gréco-romaine : la dimension d'« honnête homme " passait par cette connaissance de l'Antiquité et certains officiers éprouvaient une véritable passion pour la marine antique, passion qui s'inscrit dans un contexte d'étude et de reconstitution des marines antiques au $\mathrm{XVIII}^{\mathrm{e}}$ siècle $^{11}$. La connaissance de l'antiquité grecque demeure donc littéraire et il faut noter qu'aucune personnalité de l'Ouest ne s'intéresse vraiment à cette époque à la culture matérielle de la Grèce et de l'Italie antique, à la différence par exemple, du comte de Caylus qui partit en Italie pour visiter "les lieux, les monuments, les collections..." et parcourir les sites archéologiques ${ }^{12}$ avant de se rendre, en 1716, en Turquie. Il est vrai que ce

provinciaux, 1680-1789, Paris, La-Haye, 1978, p. 50-54; PoMIAN, Krzysztof, Collectionneurs, amateurs et curieux. Paris, Venise, XVte-XVII ${ }^{e}$ siècle, Paris, 1987, p. 248-291.

6. KerEBel, Hervé, Corseul (Côtes-d'Armor), un quartier de la ville antique. Les fouilles de Monterfile II, Paris, 2001, p. 14; NAVEAU, Jacques (dir.), Recherches sur Jublains (Mayenne) et sur la cité des Diablintes, Rennes, 1997, p. 24.

7. AUBERT, Gauthier, «À propos des débuts de l'archéologie en Bretagne (Corseul, Erquy et quelques autres lieux) ", Mémoires de la Société d'Histoire et d'Archéologie de Bretagne, LXXX, 2002, p. 459-482.

8. AUBERT, Gauthier, "Antiquaires et antiquités dans la région d'Auray dans la première moitié du XVIII" siècle ", Mémoires de la Société d'Histoire et d'Archéologie de Bretagne, LXXVIII, 2000, p. 107-121.

9. AUBERT, Gauthier, "Les élites bretonnes et la mer au milieu du XVIII ${ }^{\mathrm{e}}$ siècle, de la culture matérielle à la curiosité ", dans Guillaume, Pierre, La vie littorale, Paris, 2002, p. 129-142.

10. AUBERT, Gauthier, «Un collectionneur provincial vu par ses contemporains : le président de Robien (1698-1756) ", Annales de Bretagne, t. 105, n 4, 1998, p. 36-57 et du même auteur, Le Président de Robien, gentilhomme et savant dans la Bretagne des Lumières, Rennes, PUR, 2001.

11. Nous remercions Sylviane Llinarès, maître de conférences à l'Université de Bretagne Sud, pour ces informations. Voir CHALINE, Jean-Pierre, Sociabilité et érudition : les sociétés savantes en France : XIX ${ }^{e}-X X^{e}$ siècles, Paris, 1998, p. 182.

12. Voir en dernier lieu, AGHIon, Irène, "Le Comte de Caylus (1692-1765) ", dans LANDES, 
" collectionneur fastueux ${ }^{13}$ " appartenait à l'une des plus vieilles familles de France.

Ce sont d'ailleurs deux grandes figures de la noblesse qui étaient considérées au XIX ${ }^{\mathrm{e}}$ siècle comme ayant fondé l'archéologie bretonne : Christophe-Paul de Robien et Armand Maudet de Penhouët. Le Président de Robien était qualifié par Arthur de La Borderie de " père de l'archéologie bretonne ${ }^{14}$ ". Dans son cabinet de curiosités le passé gaulois et romain de l'Armorique est matérialisé par la présence d'antiquités "ethniques ${ }^{15}$ ", celles de l'histoire régionale. Elles y sont accompagnées d'antiquités méditerranéennes et proche-orientales, images de la double origine de notre civilisation : d'une part le monde de la bible et d'autre part le monde grécoromain. Le voisinage de ces antiquités ethniques et des autres méditerranéennes permet d'illustrer les deux centres d'intérêt fondamentaux du président de Robien (1698-1756), l'histoire régionale et l'histoire biblique. Ainsi, le président possède 179 objets antiques venant de l'Ancien monde, Égypte et Proche-Orient, et de l'Europe, ce qui représente incontestablement la plus grande collection de Bretagne. Et pour faire le lien entre ces deux centres d'intérêt, c'est la civilisation romaine qui attire surtout le président de Robien, cette civilisation dont il retrouve les traces monumentales à Corseul et à Locmariaquer ${ }^{16}$. Sur les 17 objets antiques qu'il déclare trouver beaux, 16 sont romains et la pièce la plus remarquable selon lui est une figure équestre haute de $17 \mathrm{~cm}$ : "On peut dire que c'est un chef d'œuvre de l'art. " Notons que le Président de Robien est en relation avec deux autres amateurs d'antiquités, le futur encyclopédiste Deslandes, commissaire de la marine à Brest et le maire de Nantes, Mellier, formant une sorte de petite "république curieuse ${ }^{17}$ ".

La seconde figure marquante de l'archéologie armoricaine se révèle fort différente de la première. Armand Maudet de Penhouët (1764-1839) ${ }^{18}$ participe activement à la mise en valeur du patrimoine antique qui caractérise

Christian (dir.), Les Étrusques en France. Archéologie et collections, Lattes, 2003, p. 343 et idem (dir.), Caylus, mécène du roi. Collectionner les antiquités au XVII' siècle, Paris, 2002.

13. CAVALIER, Odile, "Les réseaux méridionaux du comte de Caylus. Le marquis de Calvière et Esprit Calvet ", dans LANDEs, Christian, Les Étrusques en France..., op. cit., p. 350.

14. LA Borderie, Arthur de, Histoire de la Bretagne, Mayenne, 1972 (réed.), p. 116.

15. Pour la notion d'antiquités " ethniques ", voir Pomian, Krzysztof, " Les deux pôles de la curiosité antiquaire ", dans Laurens, Annie-France et Pomian, Krzysztof (dir.), L'anticomanie. La collection d'antiquités aux XVIII et XIX siècle, Paris, 1992, p. 59-68.

16. PirAult, Lionel, "L'agglomération antique de Locmariaquer ", Annales de Bretagne et des Pays de l'Ouest, 110, 2003, 1, p. 7-27.

17. AUBERT, Gauthier, Le Président de Robien..., op. cit., p. 175.

18. C'est d'un excellent mémoire de maîtrise de Claude BATY, "La phénicomanie ou le mythe phénicien dans l'Ouest ", soutenu en 2001 à Lorient, Université de Bretagne Sud, que nous tirons les informations sur Armand Maudet de Penhouët et sur le commandant Audemart. Voir LEVOT, Prosper-Jean, Biographie bretonne, recueil de notices sur tous les Bretons qui se sont faits un nom, soit par leurs vertus ou leurs crimes, soit dans les arts, dans les sciences, dans les lettres, dans la magistrature, dans la politique, dans la guerre etc., depuis le commencement de l'ère chrétienne jusqu'à nos jours, tome II, Vannes, 1857, p. 586 et BATY, Claude, "La phénicomanie... ", op. cit., p. 47-49. 
les débuts du XIX ${ }^{\mathrm{e}}$ siècle. Membre de l'Académie celtique et de la Société Académique de Nantes, il désire, comme le président de Robien une génération auparavant, éclairer le passé antique de l'Armorique grâce à l'étude de ses monuments. Mais plus que le passé romain, ce sont les origines qui l'intéressent, des origines prestigieuses puisque liées à l'histoire de grandes civilisations méditerranéennes antiques. Déjà des antiquaires bretons avaient établi un parallélisme entre le mégalithisme armoricain et les pyramides d'Égypte ${ }^{19}$, mais un parallélisme de nature chronologique qui avait pour but de convaincre de la haute antiquité de la civilisation armoricaine et non d'établir des correspondances entre les civilisations. À cette époque, la celtomanie commençait à toucher le milieu des antiquaires et c'était à la civilisation celte que l'on rattachait les mégalithes ${ }^{20}$. Dans ce contexte, Maudet de Penhouët présente une théorie originale : c'est la rencontre entre les premiers Celtes de Bretagne et les Phéniciens qui donne naissance à la brillante civilisation armoricaine. Ses preuves résident dans certains décors mégalithiques qu'il rapproche de l'art proche oriental ainsi que dans nombre d'objets locaux qu'il considère comme égyptiens ou d'inspiration orientale ${ }^{21}$. Enfin, il fait œuvre de linguiste en comparant la langue bretonne à la langue phénicienne. Comme on le voit, le périple d'Himilcon, navigateur phénicien connu pour avoir exploré les côtes atlantiques, inspira au XIX ${ }^{\mathrm{e}}$ siècle non seulement les auteurs romantiques mais aussi les archéologues. Le vicomte Édouard de Kersabiec (1825-1897), président de la Société archéologique de Nantes et de la Loire-Inférieure, consacre dans son essai sur l'histoire ancienne des peuplades gauloises de l'embouchure de la Loire, un long développement au port antique de Corbilo ${ }^{22}$, connu en particulier grâce à Strabon, port fondé selon lui par les Phéniciens. Cette fondation phénicienne trouva en 1872 une attestation archéologique par la découverte à Guérande ${ }^{23}$ d'une tablette en ardoise portant un texte de nature commerciale rédigé en phénicien. Cette tablette est un faux et il est important de noter qu'à ce jour, alors que plusieurs objets grecs et étrusques sont attestés en Bretagne et dans ses marges, aucun objet de type phénico-punique n'y est connu : "Les éventuelles relations de l'Armorique avec les comptoirs carthaginois des côtes lusitaniennes et galiciennes, par des voies maritimes explorées depuis longtemps par les

19. AUBERT, Gauthier, "Antiquaires et antiquités dans la région d'Auray dans la première moitié du XVIII " siècle ", Mémoires de la Société d'Histoire et d'Archéologie de Bretagne, LXXVIII, 2000, p. 107-121, en particulier p. 120.

20. VolHipac, C., "Les Gaulois à l'Académie des Inscriptions et Belles Lettres de 1701 à 1793 ", Nos ancêtres les Gaulois, Clermont-Ferrand, 1982.

21. Antiquités égyptiennes du département du Morbihan, 1812; Médailles armoricaines; présomptions qu'elles rappellent le culte de Bel, 1825.

22. SIOC'HAN DE KERSABIEC, Édouard, "Études archéologiques : Corbilon, Samnites, Vénètes, Namnètes, Bretons de la Loire ", Bulletin de la Société Archéologique de Nantes, 8,1868 , p. 288-296 et 9,1869 , p. 17-59.

23. Par le lieutenant de vaisseau Arthur Martin. Voir, Procès-verbal de la séance du 7 juillet 1874, Bulletin de la Société Archéologique de Nantes et de la Loire-Inférieure, 1874, p. 160 . 
marins gaditains, n'ont par ailleurs laissé aucune trace archéologique dans la région ${ }^{24}$. "Le voyage d'Himilcon, celui de Pythéas, les îles Cassitérides, le port de Corbilo alimentent les romans archéologiques. Des céramiques locales, protohistoriques et gallo-romaines sont considérées comme phéniciennes ou étrusques au XIX ${ }^{\mathrm{e}}$ siècle et quand sont visibles dans l'Ouest les premières collections de vases grecs et étrusques constituées en Italie et en Grèce, les érudits ne peuvent s'empêcher de les comparer aux vases régionaux qu'ils considèrent comme des imitations de l'art céramique grec ou étrusque ${ }^{25}$ :

«La persistance des types est tellement remarquable que nous sommes souvent embarrassés pour déterminer si un vase fabriqué dans nos contrées, au bord de l'océan, est grec ou ne l'est pas. Le vaste emporion de l'embouchure de la Loire, le Corbilon de Pythéas, a dû être un immense entrepôt d'où les vases grecs et les vases phéniciens se répandirent dans l'Armorique ${ }^{26}$."

La thèse la plus courue au XIX ${ }^{\mathrm{e}}$ siècle était donc la fréquentation assidue de nos côtes par les Grecs, Etrusques et Phéniciens qui étaient censés avoir influencé profondément les Gaulois armoricains et même avoir entraîné la fondation de Nantes quelques temps voire même bien avant celle de Marseille : "Ainsi, l'acte de naissance de Nantes la ferait apparaître entre -800 et -600, bien avant l'arrivée des Phocéens à Massalia en -600 ${ }^{27}$." Notons que nous avons l'attestation de découvertes de vases phéniciens, en fait d'époque romaine, sur les côtes atlantiques jusque dans les années $1930^{28}$ et qu'un ouvrage datant de 1986, La Bretagne avant Nominoë, reprend la thèse des rapports étroits entre l'Armorique et de grandes civilisations méditerranéennes mais en inversant radicalement les données. Son auteur pense apporter les preuves matérielles qu'Ulysse a débarqué dans l'embouchure de la rivière d'Etel et que, accompagnant les " peuples de la mer ", ce sont des pirates venus de l'Armorique qui ont fondé la civilisation étrusque : "[...] Il existe au musée étrusque de la Villa-Giulia à Rome un ensemble de petits bustes en terre cuite dont les visages pleins

24. GALliou, Patrick, "Importations méditerranéennes à l'âge du Fer ", Atlas d'Histoire de la Bretagne, Morlaix, Skol Vreizh, 2002, p. 40.

25 . Nous sommes à l'époque où les vases grecs découverts en Italie sont considérés comme étrusques : LisSARRAGUE, François, "Les vases étrusques du Comte de Caylus ", dans LANDEs, Christian, Les Étrusques en France..., op. cit., p. 361-365.

26. Parenteau, Fortuné, Catalogue du musée départemental d'archéologie de Nantes et de la Loire-Inférieure, Nantes, 1869, p. 19.

27. Histoire de Nantes, Paris, 1977, p. 29

28. Le docteur Marcel Baudoin, secrétaire général de la Société préhistorique française en 1904, défendait la thèse de l'origine phénicienne de sa ville natale : Saint-Gilles-Croixde-Vie et son ami, le colonel Paul Balagny, se fit principalement remarquer, au sein de la Société archéologique de Nantes, par un article consacré à l'activité des Phéniciens en Loire-Inférieure. Voir BALAgnY, Paul, " Les Phéniciens sur les côtes de la Loire-Inférieure et aux îles Cassitérides ", Bulletin de la Société Archéologique de Nantes et de la LoireInférieure, 73, 1933, p. 28-79; idem, " Notice sur la vie et les œuvres du Dr Marcel Baudoin ", Bulletin de la Société Archéologique de Nantes et de la Loire-Inférieure, 1941, p. 5 et BATY, Cl. «La phénicomanie », op. cit., p. 52-54. 
de vie et d'expression ressemblent d'une façon frappante à ceux des paysans de l'Ouest de la France ${ }^{29}$."

\section{La constitution des collections au $\mathrm{XIX}^{\mathrm{e}}$ siècle}

Nous avons vu que le Président de Robien ne s'intéressait guère aux questions de style et d'époque et préférait aux grandes œuvres d'arts les petits objets illustrant les cultes antiques. Au début du XIX ${ }^{\mathrm{e}}$ siècle, le beau antique est devenu une référence incontournable et les ouvrages de Winckelmann ont formé le goût de quelques érudits de l'Ouest. Dans ce contexte de la mode néoclassique, c'est dans le Maine de David d'Angers que naissent les premières collections de vases grecs. Pierre-Aristide Boullet Lacroix (1805-1848) fit le "Grand Tour", voyageant en Italie et en Grèce ${ }^{30}$.

C'est à Rome où il rencontra David d'Angers, Delacroix, Ingres, qu'il fit l'achat de plusieurs vases grecs et étrusques ${ }^{31}$. Mais le personnage le plus en vue à cette époque est Lancelot Théodore Turpin de Crissé (1781$1859)^{32}$. De vieille souche aristocratique, il vécut de 1809 à 1814 comme chambellan de Joséphine et il fut, à cette occasion, au contact de nombreuses personnalités artistiques de l'époque. Il fit un premier voyage en Italie en 1807 qui fut déterminant pour la naissance de sa passion des antiquités et organisa par la suite plusieurs voyages, à Naples, à Venise qui lui permirent d'acquérir de nombreux vases de grande qualité (Figures 1, 2 et 3) et de se livrer lui-même à des fouilles ou plutôt à des parodies de fouilles archéologiques. Il ne cesse dans ses descriptions de s'extasier sur la beauté ou même sur la perfection de quelques œuvres de sa collection. Anecdote qui illustre la passion romantique qui l'attachait à ses œuvres grecques, il ramène un vase de Campanie sur ses genoux, faisant ainsi le trajet de Naples

29. Prado, Jean-Jacques La Bretagne avant Nominoë, Mayenne, 1986, p. 36-38.

30. Collet, Albert, Physionomies castrogontériennes, Château-Gontier, 1910, p. 97; FrERE, Dominique, "Le legs Boullet-Lacroix au musée d'Art et d'Histoire de Château-Gontier ", dans Vases en voyage, de la Grèce à l'Étrurie, Paris/Nantes, Somogy/musée Dobrée, 2004, p. 26.

31 . Voir sur l'atmosphère d'émulation et de création artistique qui régnait à Rome à cette époque : Maesta di Roma. D'Ingres à Degas : les artistes français à Rome 1803-1873, Rome, 2003.

32. Lesseur, Catherine, "La collection Turpin de Crissé au musée Pincé d'Angers ", Vases en voyage..., op. cit., p. 22-23. 
Figure 2 - Rython en forme de tête de lévrier apulien du musée Pincé d'Angers (cl. Dominique Frère)

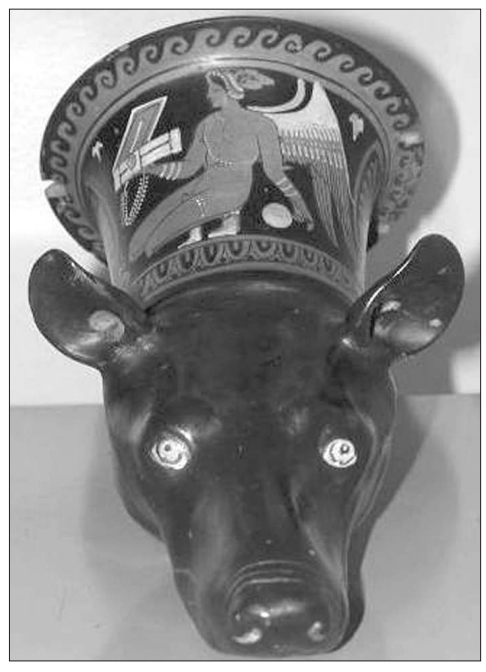

à Angers pour protéger et ne cesser d'admirer ce qu'il considère comme une perfection de l'art classique.

Mais la personnalité la plus connue de l'ouest est incontestablement le nantais Frédéric Cailliaud (1787-1869) ${ }^{33}$. Si son activité d'explorateur en Égypte est de grande notoriété, on sait moins qu'il lui était arrivé des désagréments quelques années auparavant lors de son étape napolitaine du " Grand Tour ". Dans le contexte des difficultés militaires de la fin du premier Empire, il fut forcé d'embarquer rapidement pour Constantinople en 1813, en butte à l'hostilité des Italiens, rapportant sa petite collection d'objets étrusques en bronze

Figure 3-Rython en forme de tête de bovidé apulien du musée Pincé d'Angers (cl. Dominique Frère)

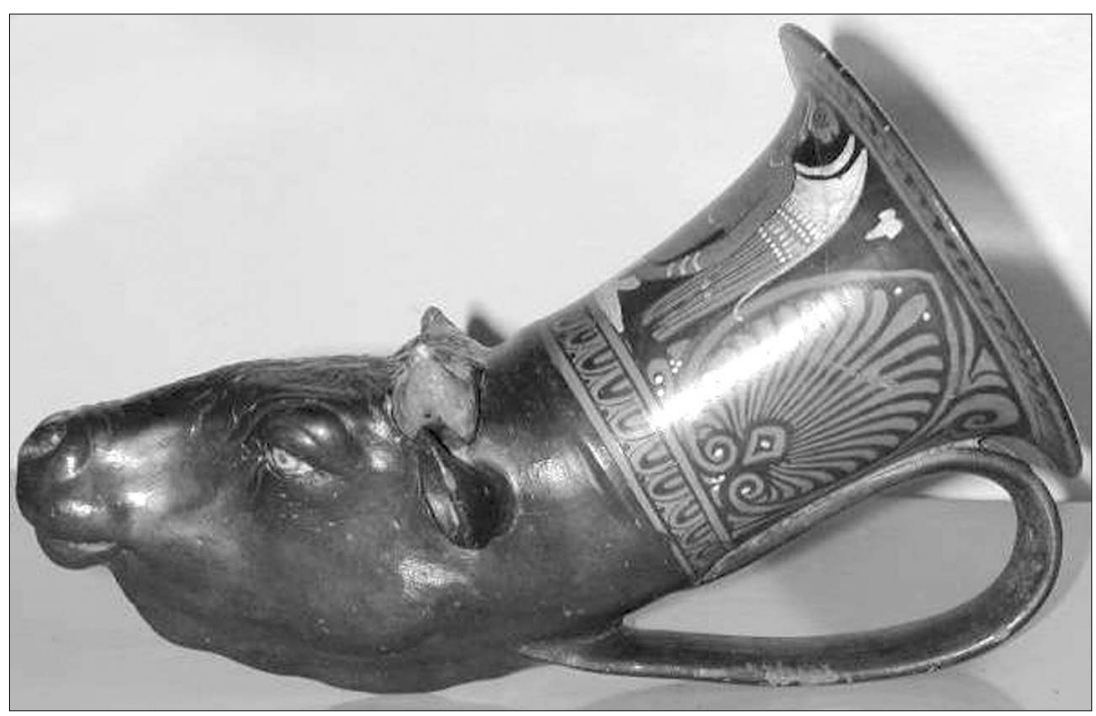

33. Voir, pour la bibliographie, CHAUVET, Marie, Frédéric Cailliaud, les aventures d'un naturaliste en Égypte et au Soudan 1815-1822, Saint-Sébastien, 1989; GRAN-AYMERICH, Évelyne, Dictionnaire biographique d'archéologie. 1798-1945, Paris, 2001, p. 130, pour l'action de Cailliaud dans le contexte de l'histoire de l'archéologie, GRAN-AYMERICH, Évelyne, Naissance de l'archéologie moderne. 1798-1945, Paris, 1998, p. 95, et pour la problématique qui nous intéresse, FIECHTER, Jean-Jacques, La Moisson des dieux. La constitution des grandes collections égyptiennes. 1815-1830, Paris, 1994. 
acquis en Campanie. Rappelons que Sir William Hamilton avait dû aussi quitter Naples dans la précipitation en 1798, devant l'arrivée des troupes du général Championnet, et avait perdu sa seconde collection antique dans le naufrage du navire de guerre qui la transportait ${ }^{34}$.

N'ayant pas connu la même mésaventure, Frédéric Cailliaud est donc à l'origine de la première collection d'antiquités publique de la ville de Nantes. Ce premier fonds sera enrichi plus tard par un lieutenant de vaisseau, Olivier de Lauriston, qui recueillit, a priori au lendemain de la guerre contre les Turcs, trente-cinq vases à Corinthe, Sicyone et Epidaure et en fit don à la société archéologique de Nantes et de la Loire-Inférieure en $1847^{35}$. On sait que nombre de militaires au XIX ${ }^{\mathrm{e}}$ siècle ont participé activement à des fouilles archéologiques et sont à l'origine de collections antiques parfois très importantes comme celle du colonel Bernard Eugène Antoine Rottiers ${ }^{36}$ dans les Pays-Bas. Le goût pour les vases étrusques était toujours affirmé dans la seconde moitié du XIX ${ }^{\mathrm{e}}$ siècle comme le prouve l'acquisition, en 1872 , de l'amiral de Kerjégu ${ }^{37}$.

De retour de la campagne du Mexique, l'amiral désirait meubler le Château-deBien-Assis qu'il venait d'acquérir dans les Côtes-d'Armor. Il charge un officier en poste dans la base militaire de Civitavecchia d'acheter des œuvres d'art pour faire du salon principal du château un petit musée. Parmi ces œuvres d'art, un superbe stamnos étrusque avec son couvercle et son support, en fait une copie napolitaine du célèbre stamnos attique du peintre du Dinos conservé au Musée national de Naples (Figure 4) ${ }^{38}$.

Figure 4 - Copie d'un stamnos attique (avec couvercle et support) de la collection de l'amiral de Kerjégu (Château de Bien-Assis) (cl. Denis Pillet)

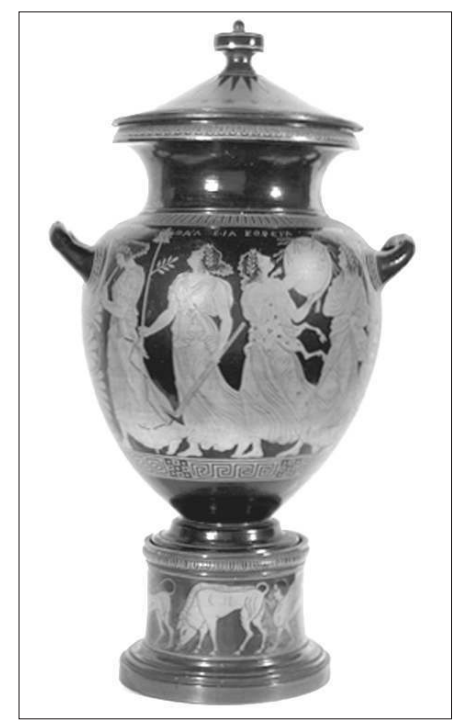

34. Smallwood, Valerie et Woodford, Suzan, Corpus Vasorum Antiquorum, The British Museum, 10, Fragments from Sir William Hamilton, Second Collection of vases recovered from the wreck of HMS Colossus, Londres, 2003, p. 11-17.

35. FreRE, Dominique, Corpus Vasorum Antiquorum, France 36, Nantes, musée Dobrée, Paris, 1997, p. 5; SANTROT, Marie-Hélène, « Un siècle et demi d'enrichissement du musée Dobrée à Nantes ", Vases en voyage, op. cit., p. 19.

36. VERREYKE, Hélène, «The Faliscan Red-Figured Stamnos of Ghent University », Bulletin Antike Beschaving, 77, 2002, p. 43-48.

37. FRERE, Dominique, "Copie d'un stamnos du musée de Naples à scènes dionysiaques ", Vases en voyage..., op. cit., p. 32-35.

38. Frere, Dominique, "Copie d'un stamnos du musée de Naples ", dans Frere, Hugot, SANTROT, Vases en voyage, op. cit., p. 32-35. 
Mais c'est seulement à la fin du XIX ${ }^{\mathrm{e}}$ siècle que trois musées, celui de Laval, celui de Nantes et celui de Vannes, s'enrichissent de collections importantes. Le commandant Audemart, membre de la société polymathique du Morbihan, se présentait lui-même comme un " soldat-archéologue ". Ami de Pierre Loti, marin et protestant, il s'était fait remarquer aux campagnes du Tonkin (1886) et de Chine (1900-1902) et avait été nommé chevalier de la légion d'honneur. Intégré en 1889 dans l'escadre de Méditerranée, le lieutenant de vaisseau Audemart s'était lié d'amitié avec le révérend père A. L. Delattre qui, depuis 1878, fouillait des tombeaux puniques ${ }^{39}$.

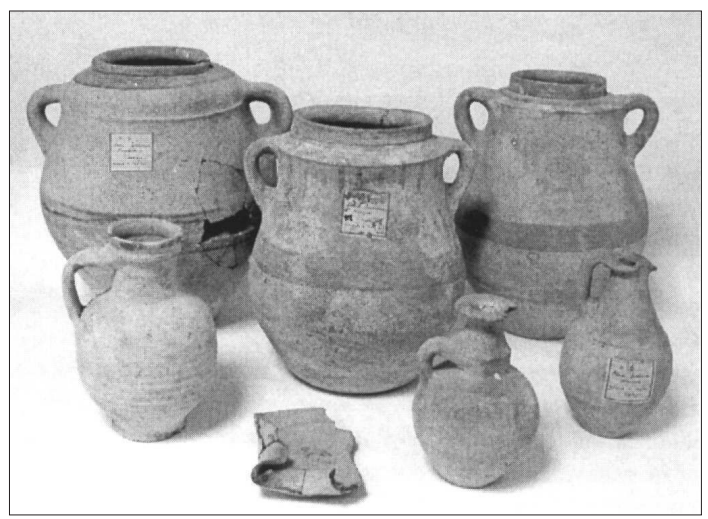

Figure 5 - Une partie de la collection du commandant Audemart (musée de la Cohue, Vannes) (cl. Christophe Le Pennec)

On sait le retentissement de ces fouilles qui dévoilaient aux Européens le passé carthaginois jusque-là surtout connu par les textes antiques. Ces fouilles donnaient lieu à de véritables cérémonies mondaines et le révérend père n'ouvrait d'ailleurs les tombeaux qu'en présence du public. Cette publi-

Figure 6 - Dessin du Commandant Audemart (Musée de la Cohue, Vannes)

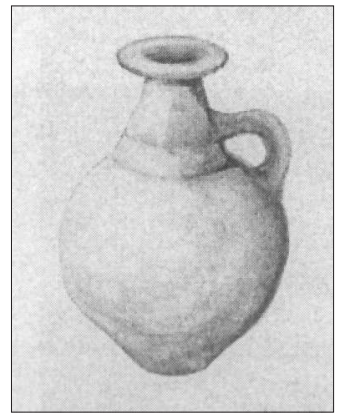
cité qu'il faisait autour de ses travaux était aussi une manière de les financer, car, en contrepartie de dons, il offrait des objets exhumés, raison pour laquelle, on en découvre à Beaufort-en-Vallée, à Nantes et à Vannes. Si ce n'est qu'à Vannes, le musée polymathique s'est vu donner le contenu d'une tombe phénico-punique (Figure 5).

Cette générosité du Révérend avait sans doute été motivée par le dynamisme et le savoir-faire d'Audemart qui avait à plusieurs reprises proposé ses services de fouilleur ainsi que ses talents de dessinateur (Figure 6).

Cette tombe a d'ailleurs fait l'objet d'une publication à laquelle a collaboré Audemart. Dernière grande figure qui participa de manière importante

39. Voir en particulier GRAN-AYMERICH, E., Dictionnaire biographique d'archéologie, op. cit., p. 211 et GrAN-AYMERICH, E., Naissance de l'archéologie moderne, op. cit., p. 244-247. 
à l'enrichissement d'un musée de l'ouest, Alphonse Robert : nommé caissier principal de la Société d'exploitation du percement du canal de Corinthe, il partit en 1868 à Isthmia d'où il ramena environ 150 vases et figurines (il en donna la moitié au musée Dobrée), présentés comme issus des fouilles faites à Hexamilia en $1885^{40}$. Fouilles ou découvertes à l'occasion des travaux du percement du canal, nous ne le savons pas.

\section{Les dépôts de l'État et les politiques des musées}

Une cartographie des collections de type grec provenant de legs et de dons nous montre qu'à la fin du XIX ${ }^{\mathrm{e}}$ siècle seuls quatre musées possédaient des collections de type grec importantes : le musée de la société polymathique à Vannes, avec un fonds de vases grecs d'origine inconnue ${ }^{41}$, le musée de Château-Gontier, le musée Dobrée de Nantes et le musée Pincé d'Angers. Le musée des Beaux-Arts de Rennes n'avait guère enrichi son fonds d'antiquités méditerranéennes ${ }^{42}$ depuis la saisie, en 1794, des antiques du cabinet de Robien ${ }^{43}$. La ville de Fontenay-le-Comte ne possédait qu'un seul vase grec, de grande qualité il est vrai, une coupe attique à figures rouges attribuée à Epiktétos ayant appartenu à la collection de Lucien Bonaparte, prince de

Figure 7 - Fragment de vase étrusque en bucchero nero du musée de Carnac (Dessin Dominique Frère)

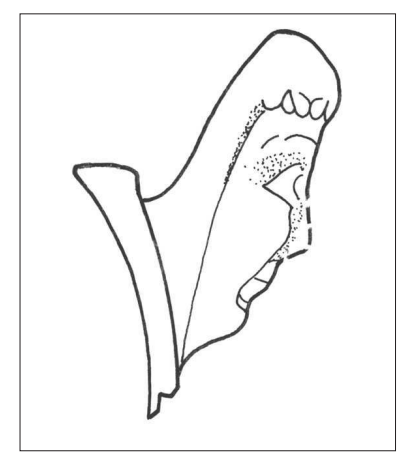
Canino ${ }^{44}$. Une seule antiquité classique est attestée au musée archéologique fondé à Carnac en 1882 par l'écossais James Miln, une tête archaïque en bucchero nero (Figure 7), tandis que la ville de Cholet n'a acquis que quatre vases par legs dans les années 1880 (Figure 8).

Le musée encyclopédique constitué par Joseph Denais à Beaufort-enVallée $^{45}$ se révèle être d'une profonde originalité : en ce qui concerne les

40. Frere, Dominique, Corpus Vasorum Antiquorum, op. cit., p. 5 et SANTROT, Marie-Hélène, "Un siècle d'enrichissement... ", op. cit., p. 20.

41. Touchefeu, Odette, Corpus Vasorum Antiquorum, France 24, musée de Limoges, musée de Vannes, Paris, 1969.

42. TouchefEu, Odette, Corpus Vasorum Antiquorum, France 29, musée de Rennes, Paris, 1979 .

43. CheVAliER, Elsa, Le Musée de Bretagne. Un musée face à son histoire, Rennes, 2001, p. 28 : " La première collection des musées de Rennes est issue des confiscations menées par l'administration révolutionnaire en 1794. Parmi ces saisies, la plus importante est celle dont sont victimes les descendants de Christophe-Paul de Robien... ".

44. FrERE, Dominique et GiRoux, Hubert, "L'identité retrouvée de la coupe d'Epictétos au Musée vendéen de Fontenay-le-Comte", Vases en voyage..., op. cit., p. 29.

45. Weygand, Sophie, "Musée d'Histoire, Histoire d'un musée, le musée Joseph-Denais à Beaufort-en-Vallée ", 303, 63, 1999, p. 40-51. 
Figure 8 - Oenochoé apulienne du musée de Cholet (cl. Étienne Lizambard)

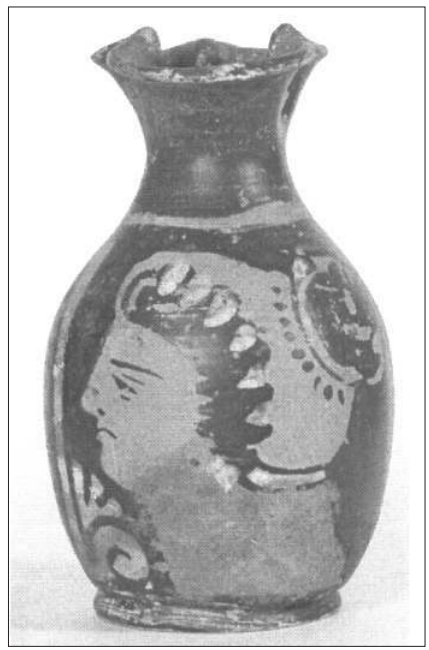

Figure 10 - Amphore attique du musée des Beaux-Arts de Brest (cl. Dominique Frère)

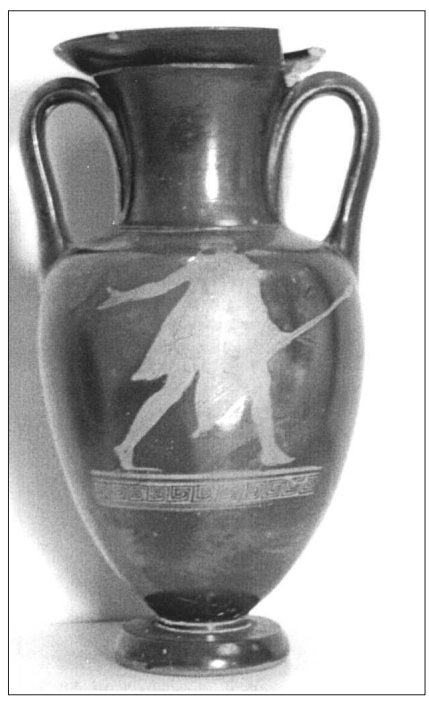

Figure 9 - Coupe en bucchero nero $d u$ musée Pincé d'Angers (dépôt du musée Guimet) (cl. Laurent Hugot)

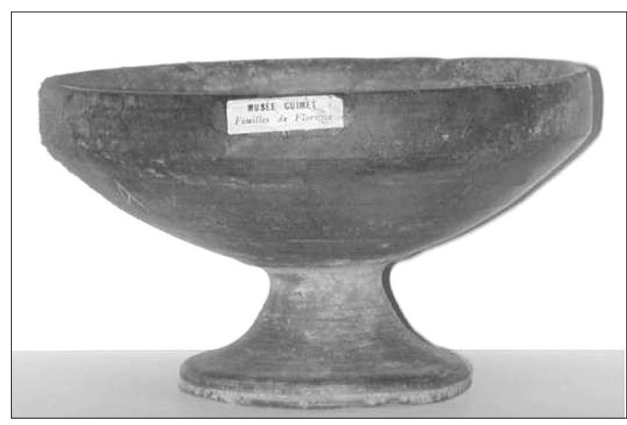

antiquités, hormis les bronzes étrusques censés avoir été découverts localement à Saint-Pierre-du-Lac, une ensemble de vases archaïques étrusques provient d'un dépôt du musée Guimet avec comme étiquette sur chaque céramique : "fouilles faites à Florence ${ }^{46}$ ". Notons que le musée Pincé d'Angers possède aussi un ensemble très semblable de vases étrusques ayant appartenu à la collection Guimet (Figure 9).

Un autre ensemble a été acheté par Joseph Denais lui-même à Tarente : il s'agit de vases et de terres cuites d'Italie du sud qui datent pour la plupart du IV ${ }^{\mathrm{e}}$ siècle av. J.-C. Enfin, le musée des Beaux-Arts de Brest reçut en 1906 un don de la Comtesse de Rodellec. Née Béatrice Charlotte Antoinette de Trobriand de Kerédern, celle qui est de vieille famille bretonne se marie en 1869 avec un riche industriel d'origine anglaise, John Burnett-Stears, qui accroît sa fortune grâce à l'affermage qu'il obtient de la ville de Brest de la distribution du gaz par becs " papillons ${ }^{47}$ ". Veuve en 1888, elle reçoit en héritage, outre la fortune importante de son mari, une collection ethnologique et archéologique formée, en ce qui concerne les antiquités méditerranéennes,

46. Frere, Dominique, "Les collections étrusques de deux musées de l'Ouest de la France : Beaufort-en-Vallée et Château-Gontier ", Annales de Bretagne, 103, 4, 1996, p. 7-24.

47. Le Télégramme du 2 février 2001. 
d'environ 80 objets (vases en céramique, terres cuites, bronzes) romains, étrusques, grecs et égyptiens (Figures 10 et 11).

Les collections grecques et étrusques d'origine privée étaient donc très inégalement distribuées dans l'Ouest, situation qui ne fut, en fait, guère réparée par les envois et dépôts de l'État en particulier dans les années 1860-1870 ${ }^{48}$.

Nous pouvons noter que dans l'Ouest, les envois de 1863 ne furent limités qu'aux grandes villes, Nantes, Rennes et Le Mans, avec l'absence notable de Brest, ce qui fait qu'un nombre important de départements fut ignoré. Cet oubli fut en partie réparé dans un second envoi, plus tardif dans musées d'Angers et de Dinan. Mais c'est surtout douze ans plus tard qu'une ville au moins de chaque département fut gratifiée d'un dépôt de l'Etat formé en grande partie de vases de la collection Campana mais aussi de céramiques mineures provenant d'autres collections. Tous ces dépôts se ressemblent donc, avec les mêmes vases des $\mathrm{VII}^{\mathrm{e}}-\mathrm{VI}^{\mathrm{e}}$ siècles (bucchero nero et céramiques de type corinthien) et du $\mathrm{IV}^{\mathrm{e}}$ siècle (vases étrusques à décor surpeint, petits vases italiotes à figures rouges). Une exception notable, celle du musée de Dinan (Figure 12).

Non seulement Dinan est la seule petite ville de l'ouest à recevoir un dépôt en 1863 mais elle est la seule aussi à bénéficier d'un autre dépôt en 1875 qui s'avère de plus être supérieur aux autres puisque y figurent des vases de qualité dont un grand stamnos étrusque à figures rouges ${ }^{49}$. Ce favoritisme dont bénéficia Dinan en 1863 et en 1875 est sans doute à rechercher dans la personnalité de Luigi Odorici, premier conservateur du musée, qui joua un rôle de premier plan dans la création du musée mais aussi dans

48. Sur la célèbre collection Campana et sa dispersion en Province, voir en particulier BRIQUEL, Dominique, "L'histoire de la collection et du dépôt Campana ", Vases en voyage..., op. cit., p. 16-18; NADALINI, Gianpaolo, «Le musée Campana : origine et formation des collections. L'organisation du musée et le problème de la restauration ", dans L'Anticomanie..., op. cit., p. 111-121; GRAN-AYMERICH, Évelyne et Jean, " La collection Campana dans les musées de province et la politique archéologique française ", dans L'Anticomanie..., op. cit., p. 123-132; NADALINI, Gianpaolo, "La collection Campana au musée Napoléon III et sa première dispersion dans les musées français (1862-1863) ", Journal des Savants, 1998, p. 183-225; GRAN-AYMERICH, E., Naissance de l'archéologie moderne, op. cit., p. 165-178.

49. Cette collection sera complétée en 1920 par un autre dépôt de l'État constitué surtout de terres cuites mais aussi d'une amphore attique à figures noires. 
Figure 12 - Plat étrusque du musée de Dinan (cl. Géraldine Guilmet)

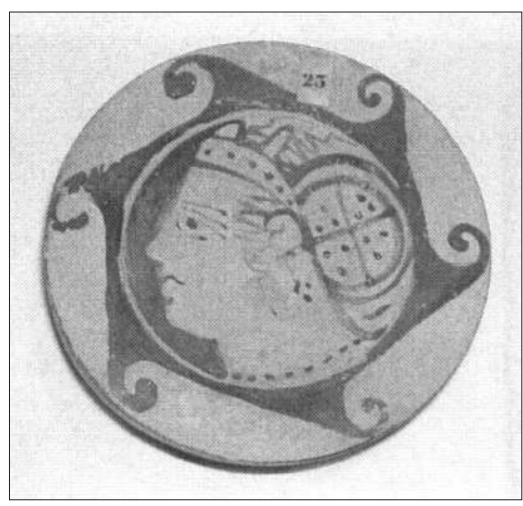

le domaine de l'histoire et de l'archéologie locale puisqu'il est l'auteur d'un ouvrage sur l'histoire de Dinan ${ }^{50}$ et fouilla à Corseul, y découvrant, parait-il, des lécythes attiques du début du ve siècle av. J.-C. ${ }^{51}$. Un autre conservateur fit beaucoup pour son musée : il s'agit de Daniel Victor Oehlert, géologue de renom qui avait décidé de compléter le dépôt fait au musée de Laval par des acquisitions de céramiques méditerranéennes ${ }^{52}$. C'est ainsi qu'il fit l'achat pendant plusieurs dizaines d'années, à Paris comme en Mayenne, de lots importants d'antiquités chypriotes, grecques et

étrusques. À la même époque, Eugène Hucher, conservateur du musée Tessé du Mans achetait aussi un lot de vases étrusques tandis que son fils, Ferdinand, en 1890, acquérait à Paris des statuettes de Tanagra ${ }^{53}$.

$\mathrm{Au} \mathrm{xx}^{\mathrm{e}}$ siècle, cette cartographie générale des collections grecques, étrusques et phéniciennes dans l'Ouest ne change guère si ce n'est que deux fonds disparaissent, celui de La Flèche lors de l'incendie de son musée en 1919 et celui de Lorient qui n'a pas été retrouvé après les bombardements de la seconde guerre mondiale. Sinon, quelques dons et legs, quelques rares dépôts surtout dans les années 1920 n'enrichissent que modestement les autres musées. Toutefois, un autre musée bénéficie d'un important dépôt de type grec : il s'agit du musée des Arts Décoratifs de Nantes qui reçoit, en 1924, 49 vases antiques dont certains de très grande qualité, du musée des Arts Décoratifs de Paris ${ }^{54}$. Ce n'est pas la nature grecque ou étrusque de ces vases qui intéressait les conservateurs de l'époque, ce n'est pas non plus

50. OdoRICI, Luigi, Recherches sur Dinan et ses environs, Dinan, 1857 (réédité en 1977); BARRAL I AltET, Xavier, « Luigi Odorici (1809-1882). Étude et sauvegarde du patrimoine historique monumental de Dinan ", Le pays de Dinan, VII, 1987, p. 167-184.

51. Cette découverte est absolument invraisemblable, premièrement parce que la céramique grecque est absente de Bretagne, deuxièmement parce que, même dans les régions françaises touchées par le commerce grec, les lécythes sont très rares si ce n'est absents, et enfin parce que les fouilles de Corseul n'ont jamais révélé d'importations méditerranéennes pré-romaines.

52. FRERE, Dominique, "Les achats d'Oehlert pour le musée du Vieux-Château de Laval ", Vases en voyage, op. cit., p. 27-28.

53. BoELDIEU-TREVET, Jeannine, "Catalogue des vases grecs et de technique grecque des musées du Mans ", Bulletin de la Société d'Agriculture, Sciences et Arts de la Sarthe, numéro spécial, 657, 1990 (paru en 1991), p. 18-19; THAURE, Marianne, " La collection didactique du musée de Tessé au Mans ", Vases en voyage, op. cit., p. 24.

54. Frere, Dominique, Corpus Vasorum Antiquorum, op. cit., p. 6 et SANTrot, Marie-Hélène, "Un siècle d'enrichissement... ", op. cit., p. 21. 
leur dimension d'objets historiques, puisque les courriers échangés entre conservateurs montrent qu'ils étaient considérés comme faux, mais d'abord leurs qualités décoratives et pédagogiques.

À la différence de ce qui a pu se passer ailleurs, la seconde guerre mondiale n'eut pas de conséquences dramatiques sur l'intégrité des collections, hormis le cas de Lorient. Les objets en céramique et en métal, pour la plupart de taille raisonnable, ne posent en effet guère de problèmes majeurs concernant leur transfert, même dans l'urgence. Pour la plupart du temps stockés dans des caisses en bois et protégés par de la paille ou du papier journal, les vases, terres cuites et bronzes, furent mis à l'abri dans des caves dès le début du conflit et, à la différence d'autres collections, ils ne furent pas convoités, que ce soit pour des raisons idéologiques ou financières, par l'occupant allemand ${ }^{55}$. Seule la destinée de la collection lorientaise demeure à ce jour énigmatique. Le dépôt de 1875 a-t-il disparu dans les bombardements ou a-t-il été transféré dans un endroit qui nous est inconnu, nous ne le savons pas. Du moins pas encore, car nous espérons recueillir des témoignages nous permettant de faire la lumière sur la disparition ou sur le lieu de conservation actuel de cette collection.

Notons que les musées reçoivent toujours des dons : ainsi à l'occasion de l'exposition "Vases en voyage ", les descendants du nantais Louis Robert ont donné au musée Dobrée quelques pièces qu'ils avaient toujours en leur possession (la moitié de la collection), en souvenir de leur ancêtre. De même des collections privées sont connues dans différentes villes de l'Ouest, certaines avec des objets de très grande valeur scientifique et artistique et d'autres avec des contextes archéologiques précis. L'étude ces collections privées et de l'histoire de leurs constitutions représente de même un travail de longue haleine qui n'en est qu'à ses balbutiements.

Ainsi, dans toute cette histoire, les vases de type grec n'ont cessé de changer de statut : d'images édifiantes de l'Antiquité, à l'idéal même du beau artistique, ils sont passés par le statut d'objets archéologiques pour acquérir dans les années 1920 celui d'objets décoratifs. Et actuellement, quelle place occupent ces collections dans le patrimoine muséologique de l'Ouest? Elles ne sont présentées que dans les musées des grandes villes hormis Brest. Dans les petites et moyennes villes, elles demeurent dans les réserves, les musées souvent consacrés à l'histoire locale, comme l'indiquent leurs noms (musée breton, musée vendéen), ne pouvant guère consacrer un espace thématique à des objets considérés comme " exotiques ${ }^{56}$ ". Toutefois, les choses évoluent puisqu'en différents lieux les conservateurs comme les élus souhaitent mettre en valeur ce qu'ils considèrent comme un patrimoine historique et une réflexion se fait jour, au moment où divers

55. Notons pour l'ouest l'intérêt des Allemands pour les objets archéologiques provenant de Carnac. Une collection prit la route de Berlin où elle disparut dans les bombardements.

56. Allemand-Cosneau, Claude, " Histoire de musées », 303, XXIII, 1989, p. 20-41. 
musées sont en restructuration ou en construction, pour statuer sur la place de ces collections archéologiques non locales dans les futures présentations.

\section{Répartition quantitative des collections}

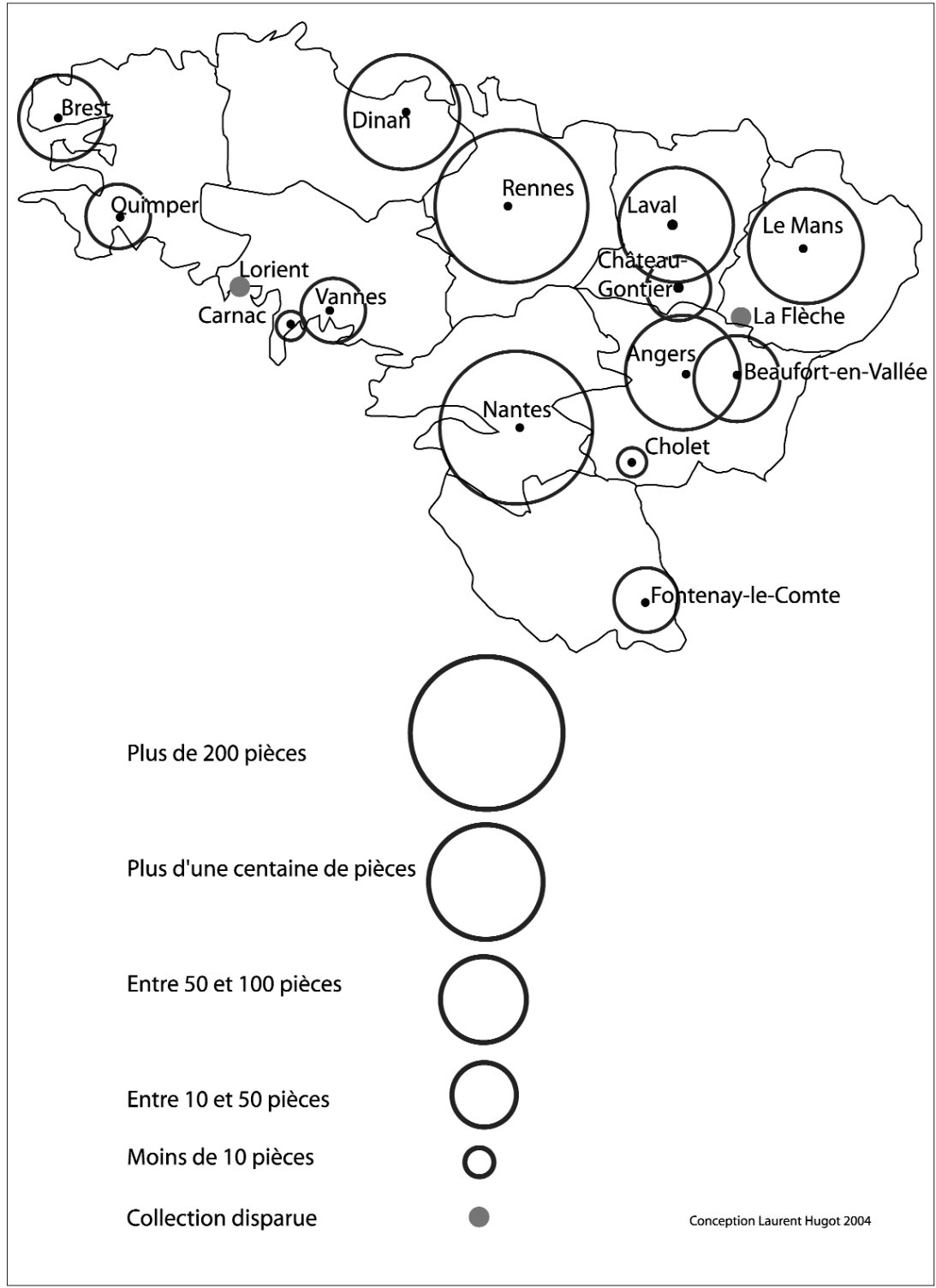




\section{Les collections de type grec en Bretagne et Pays de la Loire}

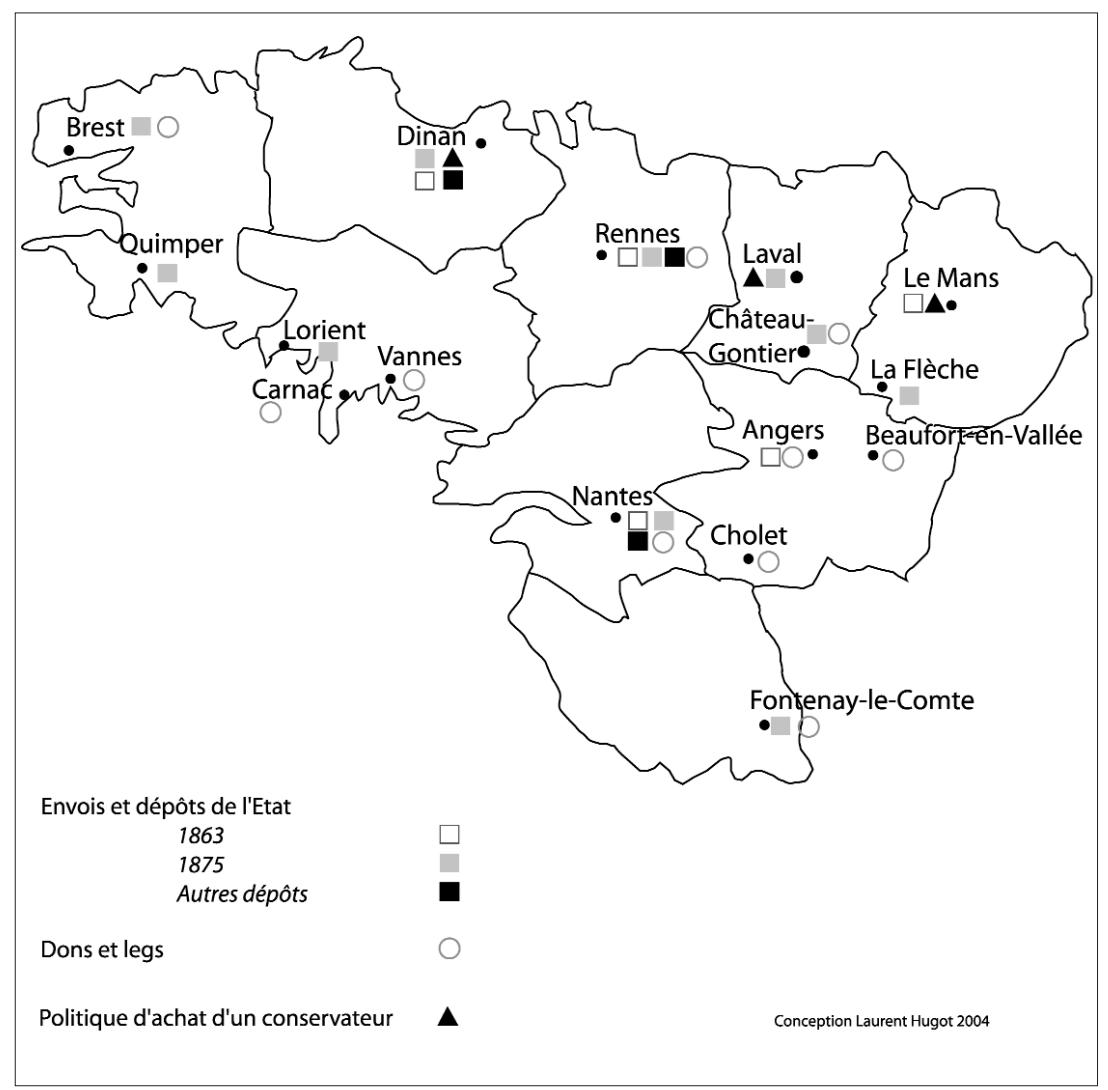

Nous tenons à adresser un chaleureux remerciement à tous les conservateurs qui accueillent les chercheurs et leur permettent de réaliser leurs travaux dans les meilleures conditions et en particulier : Françoise Daniel (musée des Beaux-Arts de Brest), Marie-Françoise Le Saux (musée de la Cohue de Vannes), Catherine Lesseur (musée Pincé d'Angers), Brigitte Richard et Géraldine Guilmet (musée de Dinan), Sophie Weygand (conseil général de Maine-et-Loire).

Nous sommes de même reconnaissants à Laurent Hugot qui nous a accompagné dans nombre de pérégrinations dans les musées et qui a réalisé les cartes du présent article et Marianne Hamiaux (musée du Louvre) qui nous a donné une aide décisive concernant l'historique des dépôts dans l'Ouest de la France. 


\section{RESUME}

La mode de l'anticomanie, apparue dans une grande partie de l'Europe dans la première moitié du $\mathrm{XVIII}^{\mathrm{e}}$ siècle, est à l'origine de la constitution de nombreuses collections privées qui, depuis la fin du $\mathrm{XIX}^{\mathrm{e}}$ siècle, ont peu à peu intégré les musées. L'Ouest de la France n'a pas échappé à cette mode et ce patrimoine constitue aujourd'hui les fonds d'archéologie méditerranéenne (Égypte, Grèce, Étrurie, Phénicie) de la plupart de nos musées. Grâce à une importante collaboration entre universitaires et conservateurs de musée, ces importantes collections sont aujourd'hui " redécouvertes " grâce à de nombreuses publications et manifestations culturelles.

\section{ABSTRACT}

Fashion for antiquities appeared in most of Europe in the first half of the $18^{\text {th }}$ century. Many private collections were then gathered in cabinets of curiosity. Since the end of the $19^{\text {th }}$ century, those collections have progressively joined museums. Western France have not escaped this fashion: today this inheritance constitutes the collections of Mediterranean archaeology (Egypt, Greece, Etruria, Phenicia) of most museums. Thanks to an important work of collaboration between academics and curators of museums, they are today "rediscovered" through many publications and cultural events. 